New approaches: A recent controlled study reported that mycophenolate mofetil is equally effective to a regimen of oral cyclophosphamide and azathioprine used sequentially in patients with proliferative lupus nephritis However, in this study follow up was short, patients had relatively mild disease and patients with high- risk factors were not included. A controlled study comparing mycophenolate mofetil to pulse cyclophosphamide is in progress. Other investigations explore the therapeutic potential of high-dose, immunoablative cyclophosphamide therapy alone or in combination with autologous stem cell transplantation, low-dose cyclophosphamide in combination with nucleoside analogues, or biologic response modifiers. High- dose cyclophosphamide or combinations of low-doses with fludarabine may result in profound bone marrow and immune suppression. Combinations of cyclophosphamide with biologic response modifiers have shown encouraging results in preclinical animal studies and may provide a major breakthrough in the treatment of severe lupus, similar to the introduction of cytotoxic agents a few decades ago.

\section{SP0103 CHRONIC RENAL FAILURE IN SYSTEMIC RHEUMATIC DISEASES}

V Tesar. 1st Department of Medicine, Charles University, Prague 2, Czech Republic

10.1136/annrheumdis-2001.46

Prevalence of end-stage chronic renal failure (ESRF) in patients with systemic lupus erythematosus (SLE) and lupus nephritis varies considerably, supposedly due to not completely comparable groups of patients and different modes of treatment, but it may be estimated that about $15-20 \%$ of patients with lupus nephritis ultimately reach ESRF. This proportion is probably similar in adults and children, but may be substantially higher in African Americans ( $43 \%$ in one study). Rapid progression to ESRF during the first year of follow-up is uncommon and may occur in some patients with active multisystem disease, more than half of patients with lupus nephritis ultimately reaching ESRF do so in more than 5 years. Chronic renal failure may be reversible in about $20 \%$ of patients with lupus nephritis starting dialysis treatment. Although the mechanisms of this very high reversibility of renal failure in lupus nephritis remain unclear, immunosuppressive treatment does not seem to be of utmost importance and other factors (e.g. hypertension, renal vasoconstriction, endothelial dysfunction, etc.) may play a role. The view that the activity of SLE remits when lupus nephritis progresses to ESRF (so called lupus burn-out) is probably no longer substantiated and according to the Dutch study $71 \%$ of patients with lupus nephritis progressing to ESRF had flares with multisystem involvement in the last 4 years before the initiation of dialysis treatment. Lupus nephritis may represent between 1-4\% of all patients treated by renal replacement therapy, their mean age is about 30 and males are more represented than in unselected SLE population. Estimated 5-year survival during hemodialysis treatment is between $81-89 \%$ and is at least comparable to other dialysis patients. Rather surprisingly, 5- and 10-year survival of patients with lupus nephritis treated by renal replacement therapy is not different from the survival of patients not progressing to ESRF. Extrarenal activity of SLE seems to be lower in patients treated by hemodialysis, but some extrarenal activity may be present in bout $2 / 3$ of dialyzed patients. Vascular access thrombosis may be much more frequent in dialyzed patients with lupus nephritis compared to other causes of ESRF, the role of antiphospholipid antibodies is suspected, but not firmly substantiated. Experience with peritoneal dialysis in patients with lupus nephritis is limited and the potential risk of infectious complications is of major concern. Renal transplantation results in relatively high risk of not surviving in the first year after transplantation (5-15\% mainly due to infectious complications. 5-year cadaveric graft survival is about $60 \%$ and renal transplantation probably does not result in higher 5 -year survival than hemodialysis. Recurrence of lupus nephritis in transplants is rare, milder and better controlled by the instituted therapy. Repeated requirement of longer dialysis treatment (usually 6-12 months) before transplantation to achieve disease quiescence does not seem to be fully substantiated.

Acute renal failure is a common presentation of ANCA-positive renal vasculitis with focal segmental necrotizing/crescentic glomerulonephritis. Dialysis dependent renal failure may occur in $24-63 \%$ of these patients (of mean age 50-60 years) according to the severity of disease and time of referral. Early immunosuppressive treatment is able to spare renal function, e.g. initial dialysis was required in 59\% and long-term dialysis in only $36 \%$ of patients from Wessex. Need for dialysis clearly confers poorer 5 -year survival (about $50 \%$ vs. $75 \%$ ). Renal transplantion is probably performed in no more than $10 \%$ of (usually younger patients with 92\% 1-year patient and 85\% 1-year graft survival. Delay in diagnosis and treatment remains to limit the therapeutic potential of current immunosuppressive treatment to improve lon-term outcome of these patients.

\section{REFERENCES}

1 Nossent JC, Swaak AJG, Berden JHM. Systemic lupus erythematosus: analysis of disease activity in 55 patients with end-stage renal failure treated with hemodialysis or continuous ambulatory peritoneal dialysis. Am J Med. 1990;89:169-74

2 Hedger N, Stevens J, Drey N, et al. Incidence and outcome of pauci-immune rapidly progressive glomerulonephritis in Wessex, UK: a 10-year retrospective study. Nephrol Dial Transplant. 2000;15:1593-9

\section{ERASS Symposium - Saturday 16 June, 10.00-11.45/Meeting Hall I}

\section{SP0106 CURRENT CONCEPTS IN ARTIFICIAL REPLACEMENT OF THE RHEUMATOID WRIST AND FINGER JOINTS}

D Herren. Department of Orthopaedic and Handsurgery, Schulthess Klinik, Zurich, Switzerland

\subsection{6/annrheumdis-2001.47}

Prosthetic replacement of wrist and finger joints is a key stone in the surgical treatment in rheumatoid arthritis. Severely destroyed joints are a source of pain and create deformities, which will severely affect the functionality of the hand. The wrist joint consists of a radiocarpal joint which provides flexion/extension and a radio-ulnar joint which allows pro-/ supination.

A mobile radiocarpal joint helps to compensate for impaired elbow and finger joint. There is a variety of wrist prosthesis on the market. Overall the results with these prosthetic devices are mixed. There is generally a high failure rate with sometimes difficult revisions situations due to missing bone stock. The Swanson silastic implant, with reasonable longtime results, might be an alternative to preserve some motion. Nevertheless arthrodesis is still the gold standard on which the current wrist implants have to be measured. On the level of the radio-ulnar joint ulnar 
head resection is still accepted as treatment of choice in most instances. Unstable distal ulnar stumps and loss of power grip raised the wish for an alternative. There is a new distal ulnar head prosthesis on the marked, which might be an possible implant for the future for selected cases.

On the level of the finger joints Swansons silastic implants, also in newer different designs, are still the implants of choice for most surgeons. High implant failure rate and the wish to go away from Silastic as implant material, motivated different authors for new implant designs and the use of newer materials. Despite these efforts a real breakthrough as well in design as in materials is still somewhat missing. Carbon fibre based materials or ceramics might have a good potential for the future.

Besides the implant design and the material, the understanding of soft tissue pathomechanics and its consequences for prosthetic replacement is raising. Ligament reconstruction and possible use of artificial ligaments might be a concept for the future.

The lecture should give an understanding of the pathomechanics of rheumatoid arthritis and its consequences for prosthetic replacement. It should give an overview of current prosthetic designs and their clinical results and provide an outlook of new concepts of prosthetic replacement and future development.

\section{SP0108 PRESENT STATUS OF TALOCRURAL ARTHROPLASTY IN RHEUMATOID ARTHRITIS}

F-W Hagena. Auguste-Viktoria-Klinik, Bad Oeynhausen, Germany

\subsection{6/annrheumdis-2001.48}

The new approach of interdisciplinary management of the multiple joint disease acknowledges the distinction between interpretation of the systemic and of the local activity. The systemic inflammatory disease may be modified or controlled by effective drug protocols. This does not prevent or exclude local inflammation or progressive alterations of various joint in the ,malignant forms of RA. In this respect rheumatoid arthritis surgery surves as local augmentation for the systemic therapy to prevent disability and/or to restore function.

An involvement of the talocrural and of the subtalar joint is reported in up to $50 \%$ in RA. At the same time the ipsilateral knee joint shows a local activity in $60 \%$ of the RA-patients.

Depending on the degree of the local activity and on the degree of deterioration the concept of rheumatoid surgery in the early phase includes the irradication of the inflamed tissue in order to reduce the progress of pain and joint damage. In the later phase related to the Larsen, Dale, Eek radiographic stages $>$ III reconstructive surgery of the talocrural joints is demanded to reduce the impairment and to restore the daily activities of the patients. To reduce pain, to stabilise and to realign the foot in neutral position ankle arthroplasties and arthrodesis are alternative procedures.

The function of the ankle joint extremity considerably influences the other joints of the lower. Stiffness and ankylosis of the ankle joint reduces the functional activity of the lower extremity. The stress distribution at the lower limb may alter the adjacent joints if the ankle joint is arthrodesed.

In former years the outcome of ankle arthroplasties has been unpredictable. The results following total replacement of the ankle using the ICLH-, TRP- or the Mayo ankle prostheses (published before 1996) reported an incidence of $22 \%$ to $75 \%$ aseptic loosening with a follow-up of 3 to 9 years. Only the experience with a follow-up of 5 years in 66 RA-patients with the modified TRP-arthroplasty reported by Pahle et al., 1987 showed good results in $83 \%$ of the patients. $1.5 \%$ of the patients had bad results after this observation time.

With new "near anatomic" designs of total ankle prostheses in a comparative study no significant difference had been observed for the OA-group $72.7 \%$ and for the RA-group $75.5 \%$ at 14 years (Kofoed, Sorensen 1998). A cumulative survival rate of the ankle replacement at 6 years of $94.3 \%$ has been reported by Schill et al., 1998. Evenmore it has been shown that in comparison to an ankle arthrodesis the total ankle replacements give significantly better pain relief, better function and a lower infection rate without development of subtalar arthrosis. Especially for patients with a multiple joint involvement in RA it is of great importance that after the total ankle arthroplasty a normal weight bearing is allowed already two weeks after implantation. In comparison to this fact ankle arthrodesis needs reduced weight bearing for at least 6 to 8 weeks.

Pain relief is achieved in more than $90 \%$ of the patients after an average follow-up time of more than 3 years. The implantation technique and the implants have been improved. Most of the available designs offer a cementless implantation of the ankle arthroplasties.

The functional results show an increased motion after total ankle replacement.

It is very important to exclude those patients with severe osteonecrosis of the talus or/and of the distal tibia to avoid early migration and the potential of early loosening.

Conclusion We like to emphasise that it seems appropriate today that in case of deterioration of the ankle joint in RA the indication for a total ankle replacement is preferable and the results are superior to an ankle arthrodesis.

\section{Biology and the destruction of cartilage/ bone - Saturday 16 June, 12.00-13.30/ Congress Hall}

\section{SP0109 HOW USEFUL ARE CONVENTIONAL DMARDS AND WHEN SHOULD BIOLOGICS, IN PARTICULAR TNF BLOCKING AGENTS, BE USED?}

L Van de Putte. Rheumatology, University Medical Centre Nijmegen, Nijmegen, The Netherlands

\subsection{6/annrheumdis-2001.49}

Rheumatoid arthritis is a chronic, systemic, inflammatory disease with polyarthritis as the most dominant clinical sign. Pharmacotherapy is, increasingly, the corner stone of treatment. Conventional drug treatment consists of NSAIDs, (combinations of) DMARDs and corticosteroids.

In recent years dramatic changes have occurred in the treatment of the disease. These can be categorised as follows:

- Early treatment: it has become clear that (irreversible) joint damage occurs relatively early in the disease course. In addition recent studies have indicated that more advanced stages of the disease are more difficult to treat.

- More and more effective DMARDs. Some of these DMARDs have a relatively fast mode of action, allowing for more adequate drug titration towards efficacy. Another interesting 